\title{
1 Scaffold-scaffold interactions regulate cell polarity in a bacterium
}

2 Wei Zhao1*, Samuel W. Duvallı*, Kimberly A. Kowallisı, Chao Zhang1, Dylan T. Tomaresı,

3 Haley N. Petitjean1, W. Seth Childers1

4 1Department of Chemistry, University of Pittsburgh, Pittsburgh, PA 15260, USA.

$5 \quad *$ Equal contributions

6

7

8 Corresponding Author:

9 W. Seth Childers

10 Chevron Science Center, Room 801

11219 Parkman Avenue

12 Pittsburgh, PA 15260

13 Phone Number: 412-624-3058

14 E-mail: wschild@pitt.edu

15

16

17

18 


\section{Abstract}

20 The localization of two biochemically distinct signaling hubs at opposite cell poles provides

21 the foundation for asymmetric cell division in Caulobacter crescentus. Here we identify an

22 interaction between the scaffolds PodJ and PopZ that regulates the assembly of the new cell

23 pole signaling complex. Time-course imaging of a mCherry-sfGFP-PopZ fluorescent timer

24 throughout the cell cycle revealed that existing PopZ resides at the old cell pole while newly

25 translated PopZ accumulates at the new cell pole. Our studies suggest that interactions between

26 PodJ and PopZ promotes the sequestration of older PopZ and robust accumulation of newl

27 PopZ at the new cell pole. Elimination of the PodJ-PopZ interaction impacts PopZ client

28 proteins, leading to chromosome segregation defects in one-third of cells. Additionally, this

29 PopZ-Pod $\mathbf{J}$ interaction is crucial for anchoring PodJ and preventing Pod $\mathrm{J}$ extracellular loss at

30 the old cell pole through unknown mechanism. Therefore, segregation of PopZ protein at the

31 old pole and recruitment of newly translated PopZ at the new pole via the PodJ scaffold ensures

32 stringent inheritance and maintenance of the polarity axis within dividing C. crescentus cells.

34 Keywords: Caulobacter crescentus; asymmetric cell division; cell polarity; scaffold proteins;

35 PodJ; PopZ; cell-cycle regulation

\section{Introduction}

38 Scaffolding proteins can direct and rewire information flow in cellular signaling

39 networks1. Through the recruitment of signaling proteins into multi-enzymatic complexes,

40 scaffolding proteins give rise to cellular functions such as cytoskeletal dynamics, cell polarity,

41 division, and morphogenesis1,2. In the bacterium Caulobacter crescentus, a set of 
42 spatiotemporally distributed scaffolding proteins are essential for the establishment and

43 maintenance of cell polarity. This underlying asymmetry enables Caulobacter crescentus to

44 divide into a motile swarmer cell and a sessile stalked cell3-5 (Figure 1).

45 Amongst the client proteins asymmetrically polarized are a set of two-component

46 signaling systems that collectively regulate the master regulator $\mathrm{Ctr} A 3,6-10$. This intricate

47 subcellular organization of CtrA regulators leads to selective CtrA phosphorylation at the new

48 swarmer pole and dephosphorylation CtrA at the old stalked cell pole (Figure 1)6,11.

49 Consequently, not only temporal12 but also spatial13 regulation of CtrA phosphorylation

50 coordinate transcription of more than 90 developmental genes14. A scaffolding factor that is

51 required for cell polarity is the protein PopZ. PopZ self-assembles as a micron-sized

52 biomolecular condensate at each cell pole13,15,16. Single-molecule tracking experiments 13, FLIP

53 studies16, and E. coli reconstitution strategies2,16,17 have shown that PopZ dynamically recruits

54 multiple distinct protein clients at each cell poles in pre-divisional cells18. However, the

55 mechanisms that enable a common scaffold to promote the formation of two compositionally

56 distinct biomolecular condensates remains unclear.

57 The new and old cell pole signaling hubs share some common clients, while others are

58 selectively recruited to each signaling hub. The PopZ scaffold promotes bipolar accumulation

59 of the histidine kinase CckA and its modulator DivL16. PopZ also serves as an attachment site

60 for the ParB-parS centromere during chromosome segregation15,18. On the other hand, the

61 histidine kinase DivJ specifically resides at the old cell pole, and the scaffolding protein SpmX

62 mediates this specific recruitment. SpmX bridges the interaction between PopZ and DivJ, and

63 can even nucleate the formation of new PopZ microdomains at ectopic poles upon

64 overexpression2. 
At the new cell pole, the scaffold proteins PopZ and PodJ play roles in polar assembly.

66 Deletion of the PodJ scaffold results in failure to recruit PleC histidine kinase to the new cell

67 pole19,20 and less monopolar accumulation of DivL at the new cell pole21. Moreover, $\Delta$ podJ

68 strains exhibited moderate loss of the localization of PopZ's client proteins at the new cell

69 pole21. Downstream, this resulted in the down-regulation of the CtrA signaling pathway21,22

70 and reduced levels of the CtrA-regulated gene PilA19,21,22. Therefore, these previous studies

71 suggest that similar to that of PopZ and SpmX at the old cell pole2, there are functional

72 interactions between the PopZ and PodJ scaffolds at the opposite cell pole. Here we

73 characterize the physical interactions between PopZ and PodJ within the new cell pole

74 microdomain, and we demonstrate that PodJ-PopZ interaction coordinates the signaling

75 transductions between their respective clients to ensure reliable asymmetric cell division.

\section{Results}

\section{Newly translated PopZ accumulates at the new cell pole}

79 A critical step in C. crescentus cell-cycle progression is the transition of PopZ from

80 being localized exclusively at the old cell pole to accumulate at both cell poles. Given that

81 PopZ scaffolds multiple cell-cycle factors 16,23, we asked how the cell-pole condensates remain

82 distinct during this change in localization patterns. One possible model is that PopZ can unbind

83 its scaffold clients at the old cell pole and self-assemble as a separate matrix at the new cell

84 pole. Alternatively, the accumulation of PopZ at the new cell pole may originate from the

85 newly translated PopZ. In support of this second model, an increase in PopZ expression is

86 observed at the same time as it is found that PopZ accumulates at the new cell pole24. We

87 approached this question with a tandem fluorescent timer by fusing PopZ to one fluorescent 
88 protein that matures rapidly (sfGFP) and one that matures substantially more slowly (mCherry)

89 (Figure 2A)25. Protein that exhibits high sfGFP fluorescence and weak mCherry fluorescence

90 represents a newly translated protein. Protein that exhibits high sfGFP and high mCherry

91 represents older protein. In past work applying this fluorescent timer approach, we

92 demonstrated that in newborn swarmer cells, newly translated SpmX-mCherry-sfGFP

93 accumulates at the old cell pole and ages as cells mature into pre-divisional cells26.

94 Time-course imaging on a synchronized $C$. crescentus population of mCherry-sfGFP-

95 PopZ revealed that the new cell pole PopZ exhibited high sfGFP but weak mCherry signals at

96 30-minutes post-synchrony. In contrast, the old cell pole contained PopZ protein displayed

97 both high sfGFP and mCherry signals (Figure 2A). At later time points in the cell cycle, 120-

98 minutes post-synchrony, both high levels of sfGFP and mCherry can be observed at the new

99 cell pole. This experiment indicated that older mCherry-sfGFP-PopZ is sequestered at the old

100 cell pole, while the new cell pole is populated with newly translated PopZ protein. It is

101 reasonable to presume that the sequestration of the old-new PopZ scaffolds may play a role in

102 preventing the homogenization of PopZ and its clients at the new and old cell pole. Since PopZ

103 subcellular localization abides by DNA occlusion mechanism27, a key question that follows is

104 what promotes the accumulation of the newly translated PopZ at the new cell pole.

105 PodJ regulates the amount of PopZ localized at the new cell pole

106 Previous studies have shown that $\mathrm{ZitP}_{28}$, TipN29, and ParA30 play redundant roles in

107 the accumulation of PopZ at the new cell pole but implicate one or more additional unknown

108 players. We hypothesized that a PopZ-PodJ scaffold-scaffold interaction may occur since only

109 PodJ could provide the recruitment capability in these players at the new cell pole19-22,31. 
111 in the $\triangle p o p Z$ strain (Figure S1A). However, we also observed an increase in cells exhibiting

112 bipolar localization (Figure S1A). This increase in PodJ bipolar accumulation could be due to

113 differences in Pod $\mathbf{J}$ protein levels or changes levels of Pod $\mathbf{J}$ proteolysis. For example, in strains

114 lacking the PodJ protease PerP, the number of cells that exhibit bipolar accumulation of PodJ

115 substantially increased (Figure S1B), consistent with past observations32. Notably, we did not

116 observe an increase in diffuse PodJ in the $\triangle p o p Z$ strain. Therefore PodJ's ability to accumulate

117 at the cell poles is independent of the PopZ scaffold.

118 We did, however, observe a 3-fold reduction of PopZ accumulation at the new cell pole

119 in the $\Delta p o d J$ versus wild-type strain (Figure 3A). Expression of sfGFP-PodJ from the

120 chromosomal xylose locus recovered the robust PopZ accumulation at the new cell pole

121 (Figure 3A). These results suggest that PodJ plays a role in regulating the amount of PopZ

122 accumulation at the new cell pole. We also observed that cells without full-length PodJ also

123 showed a decrease in total cell mCherry-PopZ intensity (Figure 3C). This suggests that deleting

124 the native podJ gene may alter PopZ transcription levels. Hence, the decreased mcherry-PopZ

125 accumulation at the new cell pole may be due to reduced expression of mCherry-PopZ or loss

126 of physical recruitment. We therefore, examined the distribution of PopZ in cells by

127 constitutive expression of mCherry-PopZ from the vanillate locus. Also, a 4-fold reduction in

128 the fraction of mCherry-PopZ signal at the new cell pole was observed in $\Delta p o d J$ compared to

129 the wild-type strain (Figure S2A, S2B). Therefore, higher levels of PopZ expression alone are

130 not capable of rescuing the loss of PopZ accumulation at the new cell pole.

131 We also performed time-lapse microscopy experiments to examine the mCherry-PopZ

132 localization throughout the cell cycle starting with a synchronized population of swarmer cells 
133 (Figure S2C). Images were acquired every minute, and kymographs were constructed to show

134 the fluorescence intensity along the cell body over time. In wild-type cells, robust mCherry-

135 PopZ foci accumulated at the new cell pole approximately 40 minutes post-synchrony (Figure.

$136 \mathrm{~S} 2 \mathrm{C}$, Movie S1). However, in a $\Delta \operatorname{podJ}$ strain, we detected significantly reduced signal at the

137 new cell pole (Figure S2C, Movie S2). Moreover, a subset of nascent swarmer cells that lacked

138 any observable PopZ focus were observed (Figure S2D). This loss of PopZ could be

139 complemented by expressing sfGFP-PodJ (Figure S2D). Amongst these swarmer cells, we

140 found $91 \%$ of cells ultimately accumulated PopZ at the correct, old cell pole (Figure S2E). We

141 observed that $9 \%$ of these cells accumulated PopZ at the new cell pole after inheriting no PopZ

142 (Figure S2E). Thus, this subpopulation of swarmer cells exhibited an abnormal switching of

143 the polarity axis.

144 This observed reduction in PopZ new cell pole accumulation mirrors loss other 145 redundant factors $\left(\mathrm{TipN}_{33}\right.$ and $\left.\mathrm{ZitP}_{28}\right)$ that play roles in promoting PopZ new cell pole 146 accumulation. This redundancy in PopZ recruitment likely reflects how deletion of podJ does 147 not result in phenotypes seen in cells with popZ deleted 15. Collectively, these results suggest 148 that the degree and the time of PopZ accumulation at the new cell pole depends on PodJ, but 149 PodJ cell pole accumulation is independent of PopZ.

$151 \quad$ PodJ deletion impacts ParB segregation in a subset of cells.

152 Past work from Brun and co-workers have shown that the PopZ client CckA exhibits 153 reduced new cell pole localization when podJ is deleted or truncated21. Another critical role of 154 PopZ is to tether the ParB/origin segregation complex at the cell poles15. The robust tethering 155 of ParB to the cell poles involves simultaneous interactions with numerous ParB/parS 
156 complexes17,34. Therefore, we investigated if the reduction of PopZ accumulation at the new

157 cell pole impacted ParB tethering. Previously, Bowman and co-workers demonstrated that

158 ParB was tethered more stably at the new cell pole than at the old cell pole after chromosome

159 segregation23. We observed that ParB-CFP was able to readily accumulate at the new cell pole,

160 while ParB-CFP foci were more mobile at both the swarmer and stalk pole, with the greater

161 change in mobility at the swarmer cell pole when cells lacking PodJ (Figure 4A). This

162 observation suggests that a PodJ mediated recruitment of PopZ impacts the dynamics of the

163 ParB/origin complex at the cell pole This close association of ParB with the cell poles is likely

164 due to the lower degree of subcellular accumulation of PopZ at the new cell pole. Alternatively,

165 it may also suggest that the Pod-PopZ interaction allosterically impacts the PopZ-ParB

166 interaction.

167 Additionally, we observed that $35 \%$ of cells displayed ParB focus detachment

168 phenotypes in the podJ deletion strain at both cell poles. In the most prevalent cases, the ParB

169 focus would translocate across the cell to the new cell pole before chromosome duplication

170 (Figure 4B). This premature centromere translocation results in the reversal of the inherited

171 cell polarity axis. In another case, we observed new and old cell pole ParB foci coalescing into

172 a single focus at the middle cell, then separating back to the cell poles (Figure 4C). Consistent

173 with the mobility analysis results (Figure 4A), these phenotypes suggest the PodJ recruitment

174 of PopZ facilitates robust PopZ-ParB chromosome tethering at the new cell pole.

175 Given that ParB also directly interacts with the cell division inhibitor protein MipZ35,

176 we examined the impact of the podJ deletion upon MipZ and FtsZ. These ParB segregation

177 defects also resulted in a less robust MipZ localization at the cell poles and a more diffuse FtsZ

178 Z-ring assembly (Figure S3A, S3B). Overall in the podJ deletion strain, cells were viable as 
179 chromosome segregation, and division processes remained mostly functional. However, PodJ's

180 interaction with PopZ seems to fine-tune chromosome segregation such that it avoids polarity

181 axis inversions.

\section{$182 \quad$ PodJ promotes bipolarization of PopZ in E. coli}

183 To determine if PodJ and PopZ interact directly, we heterologously co-expressed PopZ

184 and PodJ scaffolds in E. coli (Figure 5A, 5B). Notably, the $\gamma$-proteobacterium E. coli is highly

185 divergent from the alphaproteobacterium $C$. crescentus and does not contain any $C$. crescentus

186 polarity protein homologs. E. coli has thus been used extensively as an orthologous system for

187 testing $C$. crescentus protein-protein interactions15,16,27,28. A previous screen of PopZ

188 interaction partners indicates that PopZ and PodJ were only partially co-localized when co-

189 expressed in E. colit6 despite their co-localization in C. crescentus. This previous study utilized

190 a C-terminal fluorescent protein fusion to PodJ, while previous PodJ studies have used an N-

191 terminal fluorescent protein fusion of $\operatorname{Pod} \mathrm{J}_{32,36}$. Therefore, we hypothesized that the C-terminal

192 fluorescent protein fusion might impact PodJ localization and therefore disturb PodJ-PopZ

193 binding. To test this idea, we heterologously expressed an $\mathrm{N}$-terminal fluorescent fusion

194 protein of PodJ in E. coli. As shown in Figure 5A, YFP-PodJ exhibited readily bipolar

195 localization in about $80 \%$ of $E$. coli cells (Figure 5A, S4). PopZ accumulates at a single cell

196 pole in about $75 \%$ of cells when expressed alone, as observed in past studies 18,27 (Figure 5A,

197 S4). However, mCherry-PopZ co-localized in a bipolar pattern when co-expressed with YFP-

198 PodJ (Figure 5A, 5B). Therefore, these experiments indicated that PodJ could bipolarize PopZ

199 in E. coli (Figure 5, S4). Interestingly, this PodJ-mediated bipolarization of PopZ might be a

200 general feature of membrane-bound PopZ client proteins as SpmX2, ZitP28, and DivL16 all can 201 bipolarize PopZ in E. coli. 
PopZ-PodJ interaction is conserved amongst alphaproteobacteria

A subset of alphaproteobacteria encodes both PopZ and PodJ scaffolding proteins.

205 Notably, in the alphaproteobacteria Agrobacterium tumefaciens, past studies have

206 demonstrated a strong genetic interaction between PodJ and PopZ37,38. However, from these

207 prior studies, it remains unclear if AtPodJ and AtPopZ interact directly or indirectly. To test

208 this idea, we expressed PodJ fusion proteins from select alphaproteobacteria together with their

209 corresponding PopZ variants in E. coli (Figure 5C). Each mCherry-PopZ homolog

210 accumulated at a single cell pole when expressed alone, similar to $C c$ PopZ (Figure 5C). Each

211 YFP-PodJ variant accumulated at the cell poles, but compared to $C c$ PodJ, the variants

212 displayed heterogeneity in their subcellular localization pattern. However, in each case, we

213 observed that co-expression with PodJ results in bipolarization of PopZ (Figure 5C). These

214 experiments indicate that the interaction between PopZ and PodJ is direct and conserved

215 amongst alphaproteobacteria that contain both PopZ and PodJ.

PopZ interacts directly with PodJ's CC4-6 domain

218 To determine the PopZ binding site within PodJ, we screened the capability of PopZ to

219 bind to the library of PodJ domain deletion variants through co-expression in E. coli (Figure

220 6A, S4). We considered the following outcomes as an indication of a PopZ interaction with

221 the PodJ variants: (1) the two proteins are 100\% co-localized, and (2) the localization pattern

222 of either protein is changed after co-expression. We found that the deletion of the C-terminal

223 periplasmic domain or the intrinsically disordered PSE domain in PodJ did not disrupt the

224 PodJ-PopZ interaction (Figure 6A, Figure S4). In contrast, the deletion of the CC4-6 domain 
225 disrupted PopZ co-localization with PodJ (Figure 6A). We then expressed YFP-CC4-6 alone

226 and observed that it was diffuse through the cytoplasm in E. coli. However, it co-localized with

227 mCherry-PopZ at the cell pole when co-expressed in E. coli (Figure 6A). These data indicate

228 that coiled-coil 4-6 in PodJ is critical for co-localization with PopZ in E. coli.

229 To confirm that this PopZ-PodJ protein-protein interaction is direct, we performed in

230 vitro fluorescence polarization assays to detect PopZ-PodJ binding. In these assays, we mixed

$23116 \mu \mathrm{M}$ PopZ together with 100 nM BODIPY-PodJ CC4-6 or BODIPY-PodJ PSE fluorescently

232 labeled proteins. As shown in Figure 6B, PopZ bound to PodJ CC4-6 but did not bind to the

233 PodJ PSE construct. Both the E. coli heterologous expression assays and in vitro biochemical

234 assays show that the coiled-coil 4-6 region of PodJ is the site of interaction with PopZ.

235 PodJ-PopZ interaction regulates PopZ new pole localization and loss of PodJ from cells

236 In $C$. crescentus $\Delta p o d J$, we observed that the expression of sfGFP-PodJ $\Delta C C 4-6$ was

237 able to localize at the new cell pole (Figure 6C). One notable difference is that sfGFP-

238 PodJ $\Delta$ CC4-6 exhibited an increased mid-cell accumulation versus sfGFP-PodJ. A second

239 critical difference is that sfGFP-PodJ $\Delta$ CC4-6 recruited about 2-fold less PopZ to the new cell

240 pole than the expression of sfGFP-PodJ (Figure 6C, 6D). A comparison of PopZ cell pole

241 intensity ratio (old/new) in the wild-type strain versus the PodJ $\Delta \mathrm{CC} 4-6$ strain and the $\Delta p o d J$

242 strain shows the ratio increases in cells lacking PodJ with a functional PopZ binding site

243 (Figure 6D). Taken together, these results suggest that the PodJ CC4-6 binding site contributes

244 to PopZ accumulation at the new cell pole.

245 To our surprise, we observed that sfGFP-PodJ $\Delta$ CC4-6 foci outside of the cell,

246 specifically at the old cell pole (Figure 6E). We also observed a similar phenomenon when

247 expressing sfGFP-PodJ in popZ deletion strain (Figure 6F). One possible explanation is the 
248 formation of minicells, which have been described in previous studies of PopZ27 and SpmX 39

249 mutant strains, and SpmX overproducing cells2. Previous work from Thanbichler et al.

250 demonstrated that mini-cell formation is commonly the result of chromosome detachment

251 errors, as observed in MipZ mutant strains35. This is partially consistent with our observation

252 of increased ParB mobility at the cell poles and abnormal ParB translocation events (Figure

253 4B, 4C). However, given the role of the PopZ-PodJ interaction at the cell poles, we would

254 expect mini-cell formation to occur equally at both poles especially at the new cell pole.

255 Ebersbach et al. previously showed that minicells produced in the popZ deletion strain occur

256 exclusively at the new cell pole27. In contrast, in the popZ deletion strain we observed

257 extracellular PodJ-rich foci exclusively at the old cell pole (Figure 6D). In addition, these foci

258 were significantly smaller than mini-cells and not observable by phase in most cases. Another

259 possibility for the observed extracellular PodJ is that PodJ or a complex, including PodJ, is

260 secreted from the cell body. This could occur via the CpaC outer membrane secretion channel,

261 which remains assembled at the old cell pole after facilitating the secretion of the PilA pilin

262 protein at the new cell pole early in the cell cycle 19,40. Notably, a second factor that plays a

263 role in pilus assembly, CpaE, is recruited to the cell pole by the PodJ scaffolding protein and

264 is required for $\mathrm{CpaC}$ localization 19,40. Investigation of this process and its relevance to cell-

265 cycle regulation will require further genetic studies. Regardless of the mechanism of PodJ loss,

266 these results suggest that PopZ-PodJ interaction is critical for robust tethering of the

267 chromosome at the cell poles (Figure 4) and prevention of loss of PodJ from the cell body

268 (Figure 6).

269 Discussion 
272 an essential role in the formation of two biomolecular condensates at each cell pole13,16. Here

273 we have discovered a direct and conserved interaction between the PopZ and PodJ scaffolds

274 (Figure 6B, S5) influences the composition and the size of biomolecular condensates at the

275 new cell pole (Figure 3, S2)13. In the absence of PodJ, we observed a 3 to 4-fold reduction in

276 the amount of PopZ that localized to the new cell pole (Figure 3, S5). This reduction in new

277 cell pole localized PopZ also had an impact upon tethering of ParB to the cell poles. We

278 observed erroneous ParB translocations from the old cell pole to the new cell pole before

279 chromosome duplication in the podJ deletion strain (Figure 4B, S5). Therefore, PodJ plays a

280 role in ensuring cells inherit and maintain their polarity axis. Overall, the observed segregation

281 and division phenotypes were mild, indicating that PopZ has the ability to self-assemble at the

282 new cell pole as other redundant proteins play a role in PopZ new-pole promotion (Figure

283 S5)28,30.

A key event in C. crescentus asymmetric division is the formation of a signaling hub

285 at the new cell pole that is compositionally distinct from the old cell pole (Figure S5). Previous

286 fluorescence recovery after photobleaching (FRAP) experiments13,16 and single-molecule

287 tracking experiments 15 collectively indicate that PopZ is sequestered at the old poles for long

288 periods of time. From these past experiments, we hypothesized that PopZ accumulation at the

289 new cell pole primarily occurs through the assembly of newly translated PopZ. To distinguish

290 newly translated from older PopZ, we applied a fluorescent-timer approach. These fluorescent-

291 timer protein fusions demonstrated that newly translated protein was enriched at the new cell

292 pole (Figure 2), while old PopZ protein was sequestered mainly at the old cell pole. Thus the 
293 combination of single-molecule tracking $(<1 \mathrm{~min}) 15$, FRAP $(0-10 \mathrm{~min}) 13,16$, and fluorescent

294 timer data (>10 min) (Figure 1) allow tracking of protein over a range of timescales, and each

295 of these methods suggests that sequestration of static PopZ assemblies play a role in preventing

296 the scrambling of contents at the cell poles.

297 Super-resolution imaging of the cell poles suggests that the molecular organization is

298 well mixed at the spatial resolution of approximately $20 \mathrm{~nm} 45$. In the absence of protein-protein

299 interaction information, the PopZ-CckA-DivL and PodJ-PleC complexes could either be

300 interacting and well mixed or non-interacting and phase-separated into discrete clusters at the

301 new cell pole. Our observation of a direct-scaffold interaction between PodJ and PopZ (Figure

3023,6, S2) likely mediates placement of PleC, CckA, DivL as a well-defined signaling complex

303 in alphaproteobacteria (Figure 5). This proximity would support previously proposed models

304 in which PleC's dephosphorylation of DivK P may generate localized zones of

305 unphosphorylated DivK P 11,19 . In contrast, simple co-localization of signaling proteins at the

306 cell poles as heterogeneous clusters and without direct interactions may not overcome the rapid

307 DivK diffusion rates that generate shallow DivK P gradients across the cell 46.

308 More broadly, recent work has identified an array of scaffolds that promote the

309 organization of bacterial cytoplasm from signaling biochemistry 16,45 to RNA biochemistry 41

310 through self-assembly as biomolecular condensates. Key questions remain as to the factors that

311 promote co-assembly, phase separation, and compositional control of these bacterial

312 biomolecular condensates. Future studies will be needed to determine if PodJ can self-assemble

313 and whether it is homogenously integrated at the membrane-PopZ microdomain surface. In

314 contrast, the absence of these scaffold-scaffold interactions, and other yet to be learned

315 mechanisms, may facilitate phase separation of distinct biomolecular condensates. For 
316 example, C. crescentus contains three known spatially resolved biomolecular condensates:

317 BR-bodies involved in mRNA decay dispersed in the cell-body41, and two PopZ-mediated

318 assemblies at opposite cell poles16. System-level understanding of the bacterial cytoplasm

319 organization within these biomolecular condensates will center on understanding the breadth

320 of scaffold-scaffold interactions.

321

322 Acknowledgments. We thank Jared Schrader and Tom Mann for providing critical reviews of

323 the manuscript. We also thank Lucy Shapiro for providing critical $C$. crescentus strains that

324 supported this study. 


\section{Methods}

\section{$328 \quad$ Bacterial Strains}

329 All experiments were performed using Caulobacter crescentus NA1000 (also known as

$330 \mathrm{CB} 15 \mathrm{~N})$ and Escherichia coli BL21. E. coli BL21 was purchased from Promega. C. crescentus

331 NA1000 was a kind gift from Dr. Lucy Shapiro (Stanford University School of Medicine).

332 More strains and expression plasmids used in this study are listed in Table S1. All relevant

333 primers are given in detail in Table S2. Plasmid and strain construction are described in the

334 supplemental information. Transformations and phage transductions were carried out as

335 described 47.

\section{Growth Conditions and Inducer Concentrations}

338 C. crescentus strains were grown at $28^{\circ} \mathrm{C}$ in PYE (peptone yeast extract) or $\mathrm{M} 2 \mathrm{G}$ (minimal

339 medium supplemented with glucose) 47 . When needed, C. crescentus cells were synchronized

340 as described 48, and swarmer cells were harvested by Percoll density-gradient centrifugation. $E$.

341 coli strains used for protein purifications and microscopy experiments were grown at $37^{\circ} \mathrm{C}$ in

342 LB medium unless otherwise stated. When required, protein expression was induced by adding

$3430.002-0.5 \mathrm{mM}$ Isopropyl $\beta$-D-1-thiogalactopyranoside (IPTG) or 0.5-10 $\mathrm{mM}$ arabinose in $E$.

344 coli, and $0.003 \%-0.3 \%$ xylose or $0.05-0.5 \mathrm{mM}$ vanillic acid in C. crescentus unless otherwise

345 stated. The induction time for microscopy experiments is 2 hours in E. coli and 3 hours in $C$.

346 crescentus. Generalized CR30 phage transduction was performed as described 47. 


\section{Phase Contrast, DIC, and Epifluorescence Microscopy}

349 Cells were imaged after being immobilized on a $1.5 \%$ agarose pad containing corresponding

350 inducers when required. Phase microscopy was performed by using a Nikon Eclipse Ti-E

351 inverted microscope equipped with an Andor Ixon Ultra DU897 EMCCD camera and a Nikon

352 CFI Plan-Apochromat 100X/1.45 Oil objective. DIC (differential interference contrast)

353 microscopy was performed using the same microscope and camera but with a Nikon CFI Plan-

354 Apochromat 100X/1.45 Oil DIC objective with a Nikon DIC polarizer and slider in place. The

355 excitation source was a Lumencor SpectraX light engine. Chroma filter cube

$356 \mathrm{CFP} / Y F P / M C H R Y$ MTD TI was used to image ECFP (465/25M), EYFP (545/30M), and

357 mCherry (630/60M). Chroma filter cube DAPI/GFP/TRITC was used to image EGFP, sfGFP,

358 and mNeonGreen (515/30M). Images were collected and processed with Nikon NIS-Elements

359 AR software.

361 Time-lapse Microscopy

362 sfGFP-PodJ, mCherry-PopZ, or SpmX-mCherry were tracked using phase and fluorescence

363 microscopy. During time-lapse experiments, phase and fluorescence images were taken in 1

364 min intervals for sfGFP-PodJ, mCherry-PopZ, and SpmX-mCherry for 1-2 cell divisions $(\sim 4$

365 h). ParB-CFP fast time-lapses images were recorded every 4 minutes over 20 minutes. Long

366 ParB-CFP time-lapses were recorded every 15 minutes for 3-4 hours. The imaging system used

367 was the Nikon Eclipse Ti-E microscope equipped with an Andor Ixon Ultra DU897 EMCCD

368 camera and NIS-Elements software. C. crescentus cells with corresponding expression gene

369 were grown to the early-log phase in M2G or PYE medium (OD600 $=0.2)$, and then induced 
370 by xylose or vanillic acid for 2 hours before synchronization. Swarmer cells were isolated from

371 the culture by centrifugation $\left(20\right.$ mins at $\left.11,000 \mathrm{rpm}, 4^{\circ} \mathrm{C}\right)$ after mixture with 1 volume of

372 Percoll (GE Healthcare). The synchronized swarmer cells were pipetted onto an agarose (2\%)

373 pad containing medium with inducers and sealed with wax. NIS-Elements software was used

374 to align time-lapse images post-acquisition.

\section{ParB-CFP tracking analysis}

377 MicrobeJ49 was used to track ParB-CFP foci during fast time-lapse experiments. Predivisional

378 cells that had already segregated a ParB-CFP focus to the new cell pole were at $\mathrm{t}=0$ were

379 analyzed. Maxima were tracked, and the raw distance changes for each 4-minute difference

380 were averaged for new and old cell pole ParB-CFP foci. Averages for two separate experiments

381 were pooled and plotted. A student's t-test was used to determine statistical significance.

383 Fluorescence Intensity Profile Analysis

384 sfGFP-PodJ variants expressing mCherry-PopZ from the native PopZ promoter were imaged

385 using the above methods. After imaging, predivisional cells expressing sfGFP-PodJ variants

386 were oriented by visualization of the stalk. The average fluorescence intensity profile using

387 normalized cell length was generated using MicrobeJ49 with the new pole at 0.0 and old pole

388 at 1.0. mCherry-PopZ was made in the same way in the same strains. MipZ and FtsZ analysis

389 were performed in the same way. 


\section{$391 \quad$ Purification of PodJ and PopZ}

392 Protein expression of all PodJ variants followed the same protocol and is described in detail

393 below for PodJ (1-635). To purify the cytoplasmic portion of PodJ(1-635), Rosetta (DE3)

394 containing plasmid pwz091 was grown in 6 liters LB medium $(20 \mu \mathrm{g} / \mathrm{ml}$ chloramphenicol and

$395100 \mu \mathrm{g} / \mathrm{ml}$ ampicillin) at $37^{\circ} \mathrm{C}$. The culture was then induced at an OD600 of $0.4-0.6$ with 0.5

$396 \mathrm{mM}$ IPTG overnight at $18^{\circ} \mathrm{C}$. The cells were harvested, resuspended in the lysis buffer $(50 \mathrm{mM}$

397 Tris- $\mathrm{HCl}, 700 \mathrm{mM} \mathrm{KCl}, 20 \mathrm{mM}$ Imidazole, $0.05 \%$ dextran sulfate, $\mathrm{pH} 8.0$ ), in the presence of

398 protease inhibitor cocktail tablets without EDTA (Roche).

399 The cell suspension was lysed with three passes through an EmulsiFlex-C5 cell disruptor

400 (AVESTIN, Inc., Ottawa, Canada), and the supernatant was collected by centrifuging at 13000

$401 \mathrm{~g}$ for $30 \mathrm{~min}$ at $4^{\circ} \mathrm{C}$. Also, the insoluble cell debris was resuspended by the recovery buffer (50

$402 \mathrm{mM}$ Tris- $\mathrm{HCl}, 1000 \mathrm{mM} \mathrm{KCl}, 20 \mathrm{mM}$ Imidazole, $0.05 \%$ dextran sulfate, $\mathrm{pH} \mathrm{8.0)}$ and its

403 supernatant was collected as well as the previous centrifugation. The combined supernatants

404 were loaded onto a $5 \mathrm{ml}$ HisTraptм HP column (GE Healthcare) and purified with the ÄKTA

405 FPLC System. After washing with 10 volumes of wash buffer (50 mM Tris- $\mathrm{HCl}, 300 \mathrm{mM} \mathrm{KCl,}$

406 and $25 \mathrm{mM}$ imidazole, $\mathrm{pH} 8.0$ ), the protein was collected by elution from the system with

407 elution buffer (50 mM Tris- $\mathrm{HCl}, 300 \mathrm{mM} \mathrm{KCl}$, and $500 \mathrm{mM}$ imidazole, $\mathrm{pH}$ 8.0), and

408 concentrated to a $3 \mathrm{ml}$ volume using Amicon Centrifugal Filter Units, resulting in > 95\%

409 purity. All PodJ variants were dialyzed with a buffer containing $50 \mathrm{mM}$ Tris- $\mathrm{HCl}(\mathrm{pH} 8.0)$,

$410300 \mathrm{mM} \mathrm{KCl}$, and then aliquoted to a small volume $(100 \mu \mathrm{l})$ and kept frozen at $-80^{\circ} \mathrm{C}$ until

411 use. 
412 His-PopZ was expressed and purified the same as described 17.

\section{Fluorescence Polarization Assay}

415 To label PodJ_PSE (471-635) and PodJ_CC4-6 (250-430), we cloned a cysteine just after the

416 6X-His-tag proteins at the N-terminal of each protein. PodJ_PSE (Cys) and PodJ_CC4-6 (Cys)

417 expression and purification followed the same protocol as PodJ mentioned above. These two

418 proteins were labeled at the cysteine using thiol-reactive BODIPYTM FL N-(2-Aminoethyl)

419 Maleimide (Thermo Fisher). The proteins were mixed with 10-fold excess BODIPYTM FL N-

420 (2-Aminoethyl) Maleimide and allowed to react for 2 hours at room temperature, and the

421 unreacted dye was quenched with mercaptoethanol (5\% final concentration). The labeled

422 proteins were purified via dialysis to remove unreacted fluorescent dye ( 5 times, $500 \mathrm{ml}$ buffer,

423 and 30 mins each).

424 Fluorescence polarization binding assays were performed by mixing $100 \mathrm{nM}$ labeled proteins

425 with $0,0.25,0.5,1,2,4,8,16 \mu \mathrm{M}$ partner protein (PopZ or BSA) for 45 minutes to reach

426 binding equilibrium at the room temperature. Fluorescent proteins were excited at $470 \mathrm{~nm}$, and

427 emission polarization was measured at $530 \mathrm{~nm}$ in a UV-vis Evol 600

428 spectrophotometer (Thermo). Fluorescent polarization measurements were performed in

429 triplicates, and three independent trials were averaged with error bars representing the standard

430 deviation.

\section{Quantification and Statistical Analyses}

432 FIJI/ImageJ50, 51, and MicrobeJ 49 were used for image analysis. The number of replicates and

433 the number of cells analyzed per replicate is specified in corresponding legends. All 
434 experiments were replicated at least 2 times, and statistical comparisons were carried out using

435 GraphPad Prism with two-tailed Student's t-tests. Differences were considered to be significant

436 when $p$ values were below 0.05 . In all figures, measurements are shown as mean \pm standard

437 deviations (s.d.).

439 Kymograph Analyses

440 Kymographs of fluorescence intensity was obtained by using the built-in kymograph function

441 of MicrobeJ49. The background signal was subtracted before the kymograph analysis, and the

442 observation of stalk at the pole of $C$. crescentus cell was defined as the old pole. The

443 predivisional cell was selected as the start point in Figure 1C and Figure 3C. In Figure 1C,

444 another round of kymograph analysis was performed after the first cell division. The new pole

445 b became the old pole after cell division and another two new poles (c and $\mathbf{d}$ ) were formed.

\section{Calculation of Subcellular Co-Localization with PodJ variants}

448 To interpret the co-localization ratio in Figure 4C and Figure S2, we used strict criteria to

449 calculate how the proteins interact with the PodJ variants, i.e., (I), the localization patterns of

450 the interaction proteins are changed after co-expression. (II), the two proteins are $100 \%$ co-

451 localized at the pole (binding) or drive each other apart from the pole (dispersion). Failure to

452 meet either of these two criteria means the interaction of the two proteins is undetermined.

453 About 200 cells were analyzed for each interaction set. 


\section{References}

4561 Good, M. C., Zalatan, J. G. \& Lim, W. A. Scaffold proteins: hubs for controlling the flow of cellular information. Science 332, 680-686, doi:10.1126/science.1198701 (2011). Perez, A. M. et al. A Localized Complex of Two Protein Oligomers Controls

the Orientation of Cell Polarity. mBio 8, doi:10.1128/mBio.02238-16 (2017).

3 Lasker, K., Mann, T. H. \& Shapiro, L. An intracellular compass spatially coordinates cell cycle modules in Caulobacter crescentus. Current opinion in microbiology 33, 131-139, doi:10.1016/j.mib.2016.06.007 (2016).

4 Curtis, P. D. \& Brun, Y. V. Getting in the loop: regulation of development in Caulobacter crescentus. Microbiology and molecular biology reviews : MMBR

475 74, 13-41, doi:10.1128/MMBR.00040-09 (2010).

5 Bergé, M. \& Viollier, P. H. End-in-Sight: Cell Polarization by the Polygamic Organizer PopZ. Trends in microbiology 26, 363-375, doi:https://doi.org/10.1016/j.tim.2017.11.007 (2018).

6 Matroule, J. Y., Lam, H., Burnette, D. T. \& Jacobs-Wagner, C. Cytokinesis monitoring during development; rapid pole-to-pole shuttling of a signaling protein by localized kinase and phosphatase in Caulobacter. Cell 118, 579590, doi:10.1016/j.cell.2004.08.019 (2004).

7 Wheeler, R. T. \& Shapiro, L. Differential localization of two histidine kinases controlling bacterial cell differentiation. Molecular cell 4, 683-694 (1999).

8 Jacobs, C., Hung, D. \& Shapiro, L. Dynamic localization of a cytoplasmic signal transduction response regulator controls morphogenesis during the Caulobacter cell cycle. Proceedings of the National Academy of Sciences of the United States of America 98, 4095-4100, doi:10.1073/pnas.051609998 (2001).

9 Angelastro, P. S., Sliusarenko, O. \& Jacobs-Wagner, C. Polar localization of the CckA histidine kinase and cell cycle periodicity of the essential master regulator CtrA in Caulobacter crescentus. Journal of bacteriology 192, 539552, doi:10.1128/JB.00985-09 (2010).

10 Tsokos, C. G., Perchuk, B. S. \& Laub, M. T. A dynamic complex of signaling proteins uses polar localization to regulate cell-fate asymmetry in Caulobacter crescentus. Developmental cell 20, 329-341, doi:10.1016/j.devcel.2011.01.007 (2011).

11 Tsokos, C. G., Perchuk, B. S. \& Laub, M. T. A Dynamic Complex of Signaling Proteins Uses Polar Localization to Regulate Cell-Fate Asymmetry in Caulobacter crescentus. Developmental Cell 20, 329-341, doi:10.1016/j.devcel.2011.01.007 (2011).

12 Laub, M. T., Chen, S. L., Shapiro, L. \& McAdams, H. H. Genes directly controlled by CtrA, a master regulator of the Caulobacter cell cycle. Proceedings of the National Academy of Sciences of the United States of America 99, 4632-4637, doi:10.1073/pnas.062065699 (2002). 
Caulobacter crescentus. Nature microbiology 5, 418-429, doi:10.1038/s41564-019-0647-7 (2020).

14 Laub, M. T., Chen, S. L., Shapiro, L. \& McAdams, H. H. Genes directly controlled by CtrA, a master regulator of the Caulobacter cell cycle. Proceedings of the National Academy of Sciences of the United States of America 99, 4632-4637, doi:10.1073/pnas.062065699 (2002).

15 Bowman, G. R. et al. A polymeric protein anchors the chromosomal origin/ParB complex at a bacterial cell pole. Cell 134, 945-955, doi:10.1016/j.cell.2008.07.015 (2008).

16 Holmes, J. A. et al. Caulobacter PopZ forms an intrinsically disordered hub in organizing bacterial cell poles. Proceedings of the National Academy of Sciences of the United States of America 113, 12490-12495, doi:10.1073/pnas.1602380113 (2016).

17 Ptacin, J. L. et al. Bacterial scaffold directs pole-specific centromere segregation. Proceedings of the National Academy of Sciences of the United States of America 111, E2046-2055, doi:10.1073/pnas.1405188111 (2014).

18 Holmes, J. A. et al. Caulobacter PopZ forms an intrinsically disordered hub in organizing bacterial cell poles. Proceedings of the National Academy of Sciences, doi:10.1073/pnas.1602380113 (2016).

19 Viollier, P. H., Sternheim, N. \& Shapiro, L. Identification of a localization factor for the polar positioning of bacterial structural and regulatory proteins.

Proceedings of the National Academy of Sciences of the United States of America 99, 13831-13836, doi:10.1073/pnas.182411999 (2002).

20 Hinz, A. J., Larson, D. E., Smith, C. S. \& Brun, Y. V. The Caulobacter crescentus polar organelle development protein PodJ is differentially localized and is required for polar targeting of the PleC development regulator. Molecular microbiology 47, 929-941, doi:10.1046/j.1365-2958.2003.03349.x (2003).

21 Curtis, P. D. et al. The scaffolding and signalling functions of a localization factor impact polar development. Molecular microbiology 84, 712-735, doi:10.1111/j.1365-2958.2012.08055.x (2012).

22 Lawler, M. L., Larson, D. E., Hinz, A. J., Klein, D. \& Brun, Y. V. Dissection of functional domains of the polar localization factor PodJ in Caulobacter crescentus. Molecular microbiology 59, 301-316, doi:10.1111/j.13652958.2005.04935.x (2006).

23 Bowman, G. R. et al. Caulobacter PopZ forms a polar subdomain dictating sequential changes in pole composition and function. Molecular microbiology 76, 173-189, doi:10.1111/j.1365-2958.2010.07088.x (2010).

24 Schrader, J. M. et al. Dynamic translation regulation in Caulobacter cell cycle control. Proceedings of the National Academy of Sciences of the United States of America 113, E6859-E6867, doi:10.1073/pnas.1614795113 (2016). 25 Balleza, E., Kim, J. M. \& Cluzel, P. Systematic characterization of maturation time of fluorescent proteins in living cells. Nature methods 15, 47-51, doi:10.1038/nmeth.4509 (2018). 
54326 Schrader, J. M. et al. Dynamic translation regulation in Caulobacter cell cycle control. Proceedings of the National Academy of Sciences 113, E6859E6867, doi:10.1073/pnas.1614795113 (2016).

27 Ebersbach, G., Briegel, A., Jensen, G. J. \& Jacobs-Wagner, C. A selfassociating protein critical for chromosome attachment, division, and polar organization in caulobacter. Cell 134, 956-968, doi:10.1016/j.cell.2008.07.016 (2008).

28 Berge, M. et al. Modularity and determinants of a (bi-)polarization control system from free-living and obligate intracellular bacteria. Elife 5, doi:10.7554/eLife.20640 (2016).

29 Lam, H., Schofield, W. B. \& Jacobs-Wagner, C. A landmark protein essential for establishing and perpetuating the polarity of a bacterial cell. Cell 124, 1011-1023, doi:10.1016/j.cell.2005.12.040 (2006).

30 Laloux, G. \& Jacobs-Wagner, C. Spatiotemporal control of PopZ localization through cell cycle-coupled multimerization. The Journal of cell biology 201, 827-841, doi:10.1083/jcb.201303036 (2013).

31 Duerig, A. et al. Second messenger-mediated spatiotemporal control of protein degradation regulates bacterial cell cycle progression. Genes \& development 23, 93-104, doi:10.1101/gad.502409 (2009).

32 Chen, J. C., Viollier, P. H. \& Shapiro, L. A membrane metalloprotease participates in the sequential degradation of a Caulobacter polarity determinant. Molecular microbiology 55, 1085-1103, doi:MMI4443 [pii] 10.1111/j.1365-2958.2004.04443.x [doi] (2005).

33 Schofield, W. B., Lim, H. C. \& Jacobs-Wagner, C. Cell cycle coordination and regulation of bacterial chromosome segregation dynamics by polarly localized proteins. The EMBO journal 29, 3068-3081, doi:10.1038/emboj.2010.207 (2010).

34 Ptacin, J. L. et al. A spindle-like apparatus guides bacterial chromosome segregation. Nature cell biology 12, 791-U746, doi:10.1038/ncb2083 (2010).

35 Thanbichler, M. \& Shapiro, L. MipZ, a spatial regulator coordinating chromosome segregation with cell division in Caulobacter. Cell 126, 147-162, doi:10.1016/j.cell.2006.05.038 (2006).

36 Chen, J. C. et al. Cytokinesis signals truncation of the PodJ polarity factor by a cell cycle-regulated protease. The EMBO journal 25, 377-386, doi:10.1038/sj.emboj.7600935 (2006).

37 Anderson-Furgeson, J. C., Zupan, J. R., Grangeon, R. \& Zambryski, P. C. Loss of PodJ in Agrobacterium tumefaciens Leads to Ectopic Polar Growth, Branching, and Reduced Cell Division. Journal of bacteriology 198, 18831891, doi:10.1128/jb.00198-16 (2016).

38 Grangeon, R., Zupan, J., Jeon, Y. \& Zambryski, P. C. Loss of PopZ At activity in Agrobacterium tumefaciens by Deletion or Depletion Leads to Multiple Growth Poles, Minicells, and Growth Defects. mBio 8, doi:10.1128/mBio.01881-17 (2017).

39 Radhakrishnan, S. K., Thanbichler, M. \& Viollier, P. H. The dynamic interplay between a cell fate determinant and a lysozyme homolog drives the 
asymmetric division cycle of Caulobacter crescentus. Genes \& development 22, 212-225, doi:10.1101/gad.1601808 (2008).

40 Viollier, P. H., Sternheim, N. \& Shapiro, L. A dynamically localized histidine kinase controls the asymmetric distribution of polar pili proteins. The EMBO journal 21, 4420-4428, doi:10.1093/emboj/cdf454 (2002).

41 Al-Husini, N., Tomares, D. T., Childers, W. S. \& Schrader, J. a-proteobacterial RNA degradosomes assemble liquid-liquid phase separated RNP bodies. Molecular cell (2018).

42 Al-Husini, N. et al. BR-Bodies Provide Selectively Permeable Condensates that Stimulate mRNA Decay and Prevent Release of Decay Intermediates. Molecular cell, doi:10.1016/j.molcel.2020.04.001 (2020).

43 Monterroso, B. et al. Bacterial FtsZ protein forms phase-separated condensates with its nucleoid-associated inhibitor SImA. EMBO reports 20, doi:10.15252/embr.201845946 (2019).

44 Heinkel, F. et al. Phase separation and clustering of an ABC transporter in Mycobacterium tuberculosis. Proceedings of the National Academy of Sciences of the United States of America 116, 16326-16331, doi:10.1073/pnas.1820683116 (2019).

45 Lasker, K. et al. Selective sequestration of signalling proteins in a membraneless organelle reinforces the spatial regulation of asymmetry in Caulobacter crescentus. Nature microbiology, doi:10.1038/s41564-019-06477 (2020).

46 Tropini, C. \& Huang, K. C. Interplay between the localization and kinetics of phosphorylation in flagellar pole development of the bacterium Caulobacter crescentus. PLoS computational biology 8, e1002602, doi:10.1371/journal.pcbi.1002602 (2012).

47 Ely, B. Genetics of Caulobacter crescentus. Methods in enzymology 204, 372-384 (1991).

48 Evinger, M. \& Agabian, N. Envelope-associated nucleoid from Caulobacter crescentus stalked and swarmer cells. Journal of bacteriology 132, 294-301 (1977).

49 Ducret, A., Quardokus, E. M. \& Brun, Y. V. MicrobeJ, a tool for high throughput bacterial cell detection and quantitative analysis. Nature microbiology 1, 16077, doi:10.1038/nmicrobiol.2016.77 (2016).

50 Schindelin, J. et al. Fiji: an open-source platform for biological-image analysis. Nature methods 9, 676-682, doi:10.1038/nmeth.2019 (2012).

51 Preibisch, S., Saalfeld, S. \& Tomancak, P. Globally optimal stitching of tiled 3D microscopic image acquisitions. Bioinformatics 25, 1463-1465, doi:10.1093/bioinformatics/btp184 (2009). 


\section{Figure Legends}

630

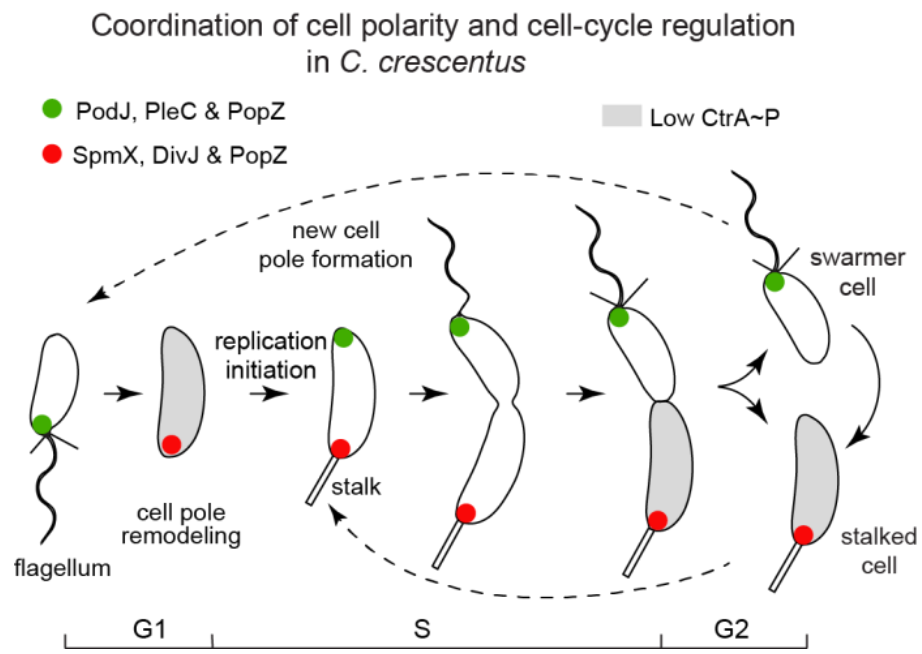

631 Figure 1: The PopZ and PodJ scaffold proteins are involved in the asymmetric

632 accumulation of signaling proteins at the new cell pole. Swarmer cells of Caulobacter

633 crescentus differentiate into stalked cells, which is associated with cell pole remodeling of a

634 PodJ-rich signaling hub (green) into a SpmX-rich signaling hub (red). At the new pole of the

635 stalked cells, a PodJ-rich signaling hub with scaffolding protein PopZ accumulates gradually

636 upon initiation of replication. Cell division results in daughter cells that involved unequal

637 inheritance of a PodJ-rich signaling hub in swarmer cell and a SpmX-rich signaling hub in 638 stalked cell.

639 


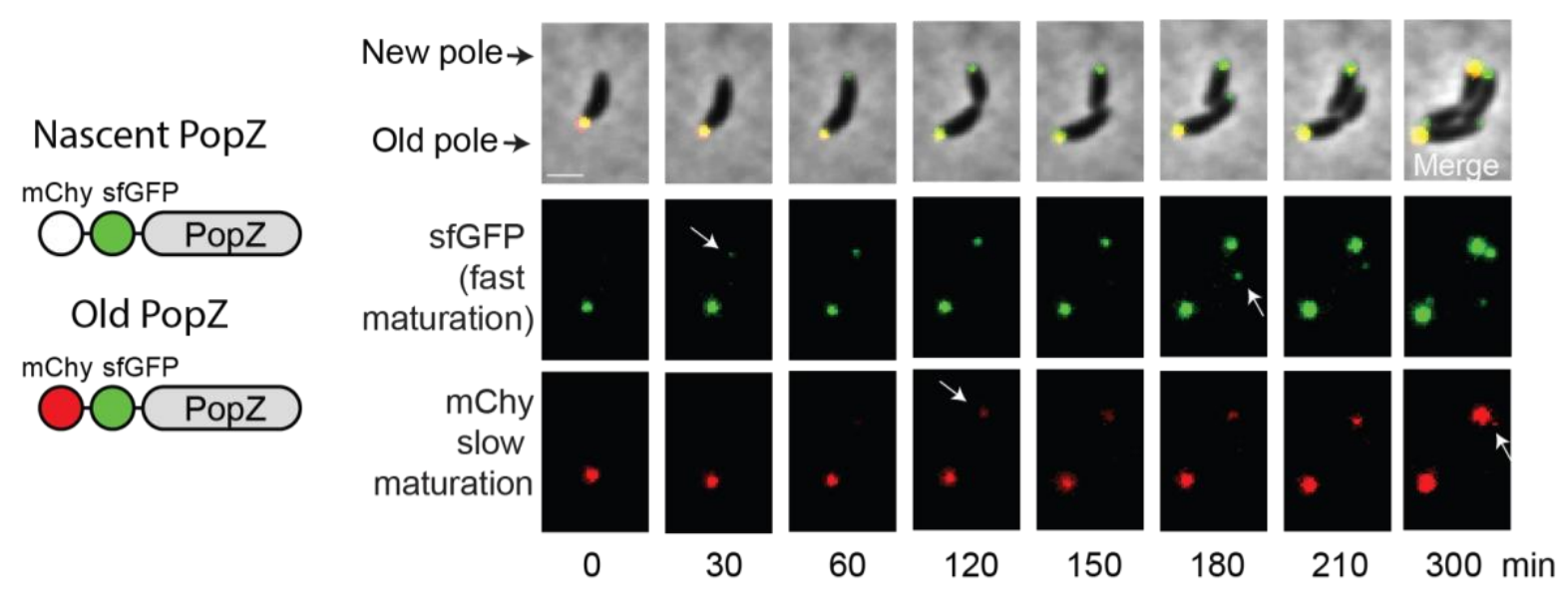

641 Figure 2: Newly translated PopZ localizes to the new cell pole in developing cells.

642 mCherry-sfGFP-PopZ is expressed under the xylose promoter in NA1000 cells. mCherry (t50

643 maturation time of $45 \mathrm{~min}$ at $32^{\circ} \mathrm{C}$ ) and sfGFP (

644 chromophores mature at different times so newly synthesized PopZ will appear green and older

645 synthesized PopZ appears as yellow. At time $0 \mathrm{~min}$, the old pole shows both green and red

646 indicating it is older yellow PopZ. At times 30-60 min a green PopZ focus appears at the

647 opposite pole. At time $120 \mathrm{~min}$ the new foci contain both green and red fluorescence, indicating

648 the subsequent maturation of the mCherry chromophore. Subsequently, in the second round of

649 cell division, a green PopZ focus appears at the new cell pole of the divided cell at time 180

650 min as the newly translated PopZ appears at the new cell pole.

651 


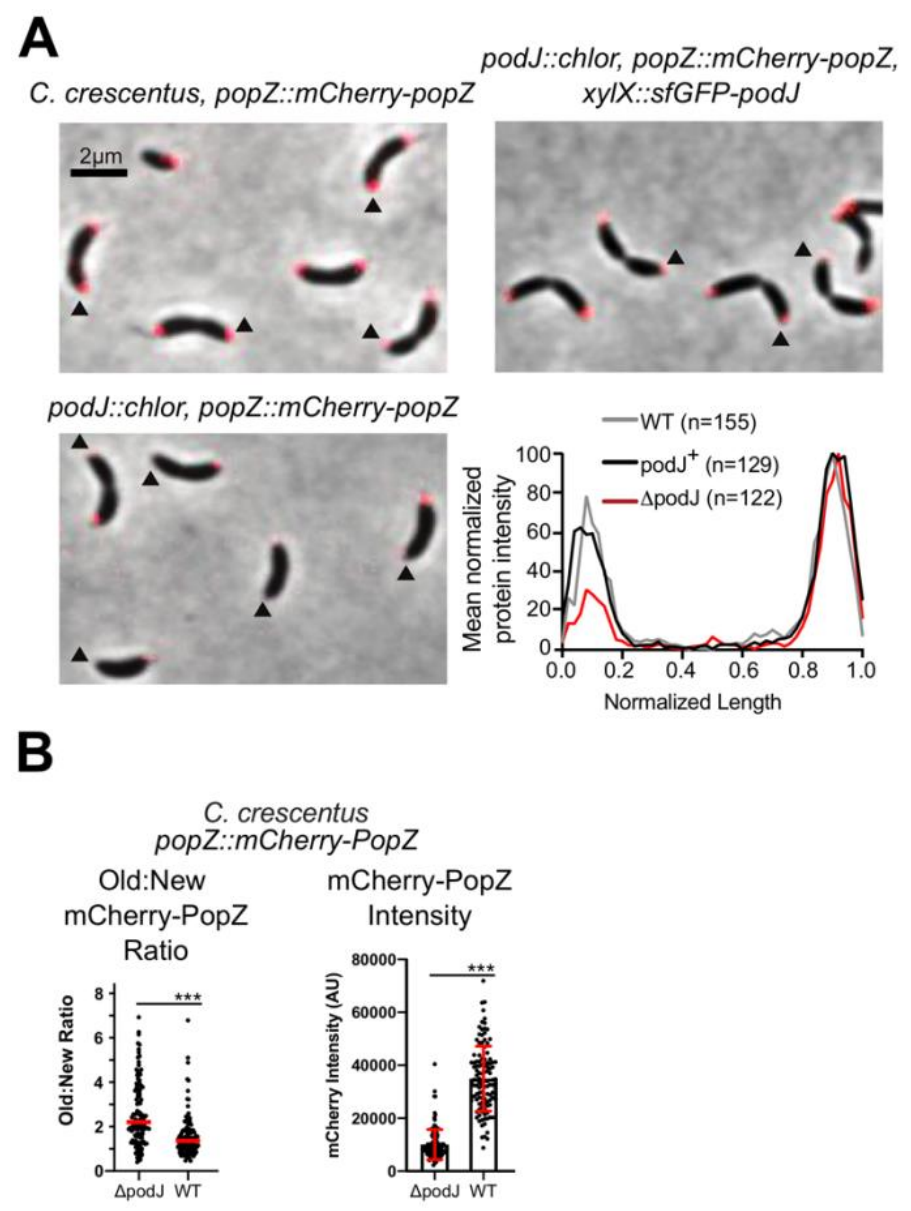

653 Figure 3: PodJ regulates PopZ assembly at the new cell pole. Analysis of the impact of

654 the $\Delta p o d J$ upon mCherry-PopZ's localization pattern in C. crescentus. The expression of the 655 sole copy popZ was induced from PopZ's native promoter in the chromosome. (A) mCherry656 PopZ localization in predivisional cells in the wild-type (bipolar) versus the podJ deletion $C$. 657 crescentus (monopolar). The quantitative analysis reveals a substantial reduction of PopZ 658 abundance at the new cell pole of $\Delta p o d J$ predivisional cells. Bars, $2 \mu \mathrm{m}$. (B) Comparison of 659 the percentage of cells displaying bipolar PopZ in wild-type and $\Delta p o d J$. Analysis of

660 Old/New cell pole ratio and total cell intensity of mCherry-PopZ in different PodJ

661 backgrounds. $* * *$ indicates $p<0.0001$. Red line indicates mean. Red bars indicated mean \pm 662 standard deviation. Statistical analysis done using student's t-test. 
A
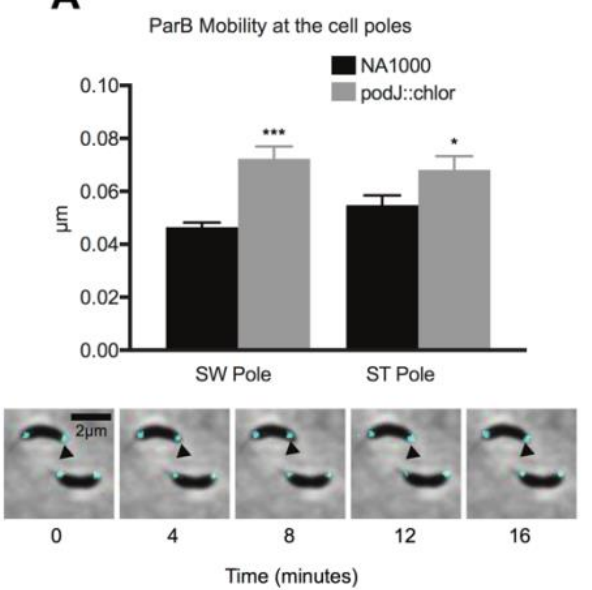

B
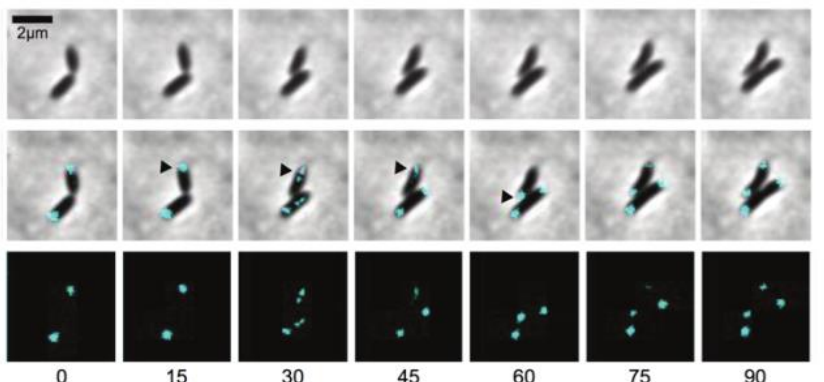

Time (minutes)

C

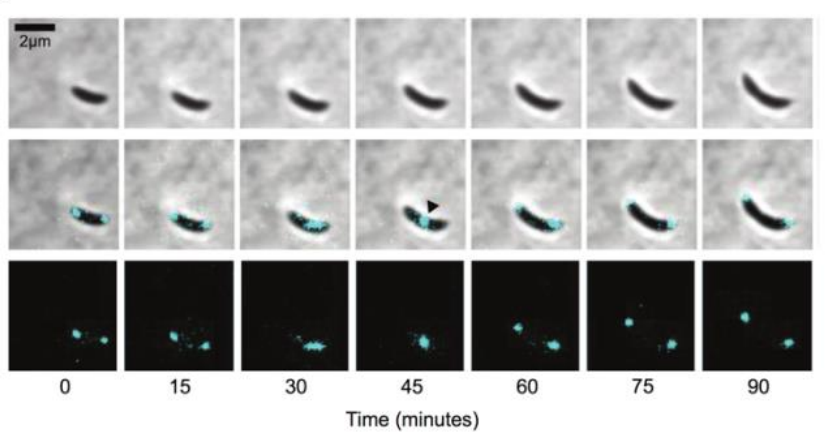

663

664 Figure 4: $C$. crescentus strains lacking PodJ exhibit chromosome segregation defects (A)

665 Analysis of ParB foci mobility at the cell poles in wild-type versus $\Delta p o d J$ strains. Cells shown

666 are $\Delta p o d J$ background. $* * *$ indicates $p<0.0001$ and * indicates $p<0.05$. Student's t-test used

667 for statistical significance. (B and C) Observed chromosome translocation defects in the $\Delta p o d J$

668 strain.

669 
A PopZ alone in E. coli

PopZ and PodJ co-expression
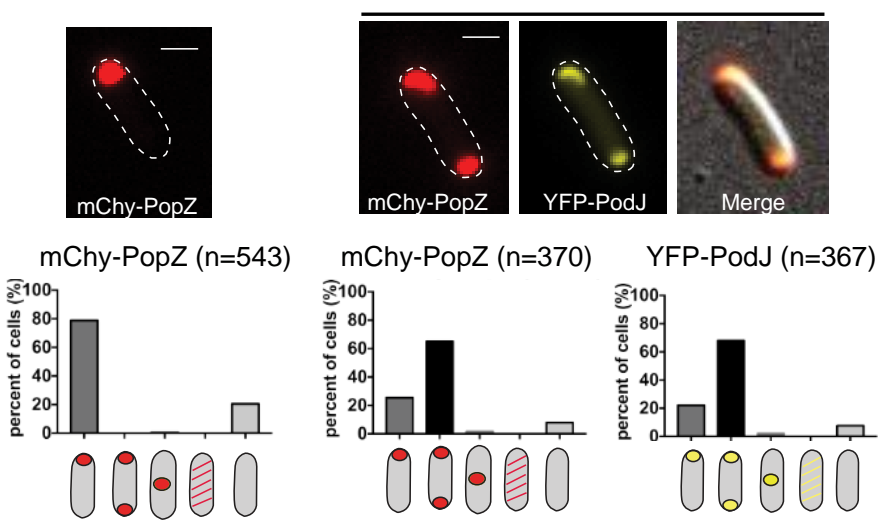

B

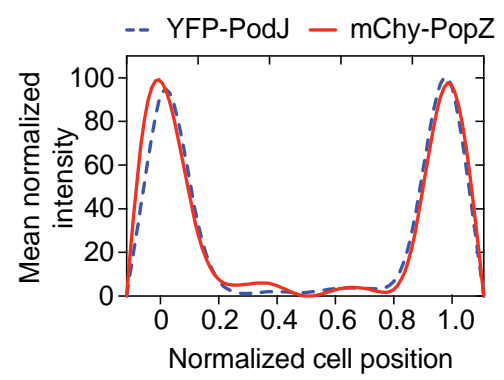

C

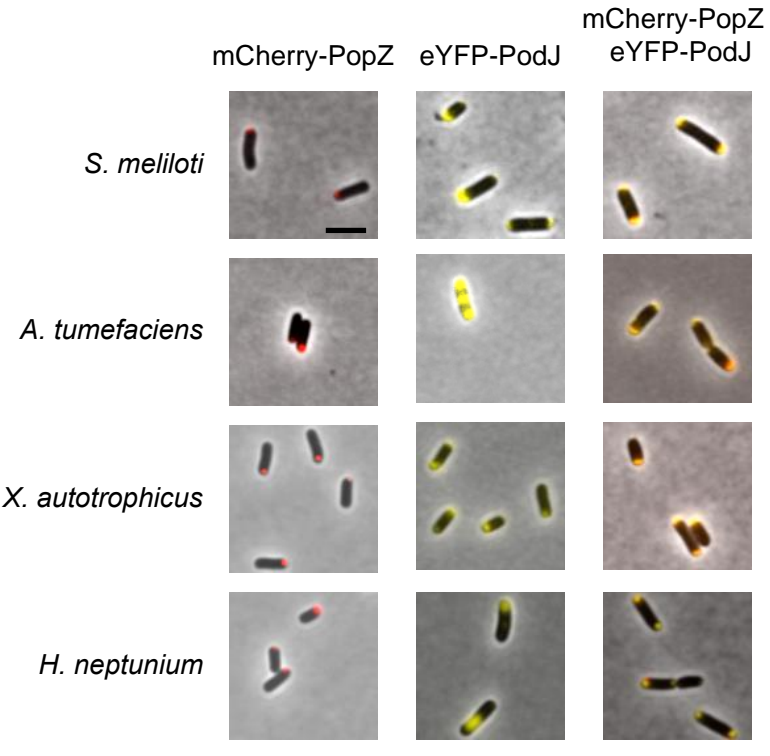

672 Figure 5: PodJ bipolarizes PopZ when expressed in E. coli, via an interaction conserved

673 across alphaproteobacteria. (A) Heterologous expression of YFP-PodJ and mCherry-PopZ

674 in E. coli. Co-expression with PodJ causes bipolar PopZ accumulation in E. coli. (B) Mean 
675 protein intensity of YFP-PodJ and mCherry-PopZ versus cell length $(\mathrm{n}=370)$. The signal

676 intensity was normalized with the highest value as $100 \%$ in each strain. (C) Co-expression of

677 PopZ-PodJ scaffold pairs from Sinhorhizobium meliloti, Agrobacterium tumefaciens,

678 Xanthobacter autotrophicus, and Hyphomonas neptunium. All PopZ homologs accumulate

679 specifically at one cell pole when expressed alone. Co-expression of PopZ together with PodJ

680 results in co-localized PopZ-PodJ bipolar localization.

681 
A
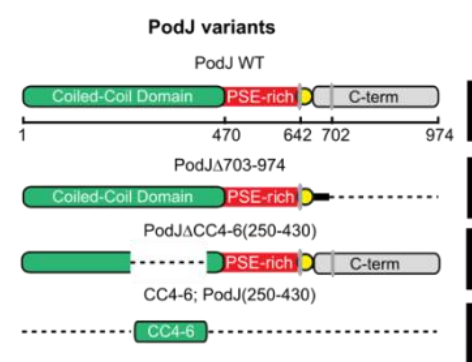

B

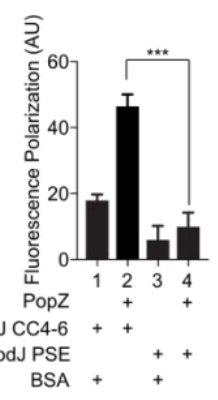

\section{C}
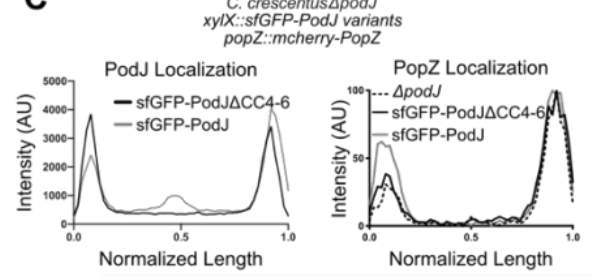

E

sfGFP-PodJACC4-6 in WT

Old:New
mCherry-PopZ
Ratio
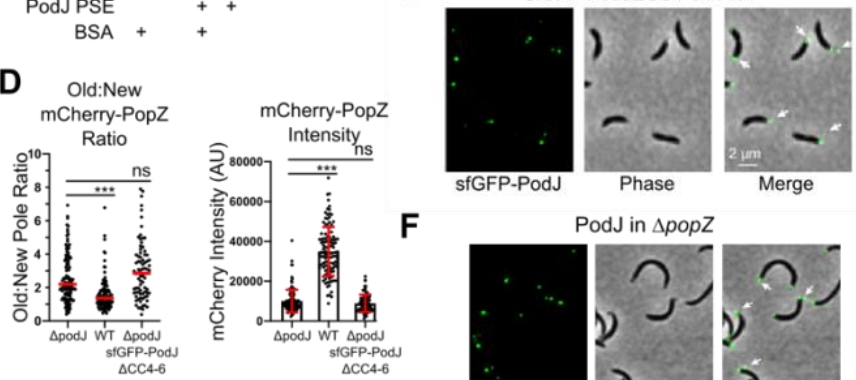

$\mathbf{F}$

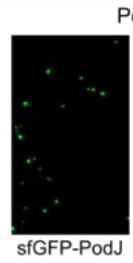

PodJ in $\triangle p o p Z$

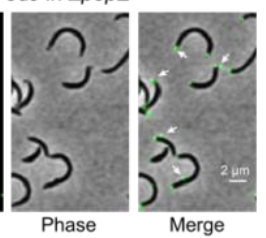

683 Figure 6: PopZ binds directly to the coiled-coil 4-6 region of PodJ. (A) Co-expression of

684 PodJ variants together with PopZ in E. coli reveals that the coiled-coil 4-6 region in PodJ is

685 necessary for the interaction with PopZ (please refer to Figure S4 for more details). (B)

686 Fluorescence polarization binding assay of the BODIPY dye-labeled PodJ_PSE or

687 PodJ $\Delta$ CC4-6 mixed with $10 \mu \mathrm{M}$ PopZ, using BSA as a negative control. PopZ binds

688 specifically to the CC4-6 domain of PodJ. However, PopZ does not bind to its PSE-rich

689 domain. (C) Fluorescent plots normalized by cell length where 0.0 is the new cell pole, 1.0 is

690 the old cell pole with the expression of sfGFP-PodJ variants from the xylose promoter in $C$.

691 crescentus. These $\Delta p o d J$ cells are also expressing mCherry-PopZ from the popZ promoter.

692 (D) Analysis of Old/New cell pole ratio and total cell intensity of mCherry-PopZ in different 
693 PodJ backgrounds. *** indicates $p<0.0001$. Red line indicates mean. Red bars indicated

694 mean \pm standard deviation. Statistical analysis done using student's t-test. (E) Loss of PodJ-

695 PopZ interaction results in stalk-pole specific foci that contain PodJ $\Delta$ CC4-6 protein. (F)

696 sfGFP-PodJ in $\triangle p o p Z$ cells. Arrows indicate sfGFP-PodJ found outside of the cell or in non-

697 polar regions of the cell. 